\title{
Erratum to: Novel Use of a Dektak 150 Surface Profiler Unmasks Differences in Resorption Pit Profiles Between Control and Charcot Patient Osteoclasts
}

\author{
Nina L. Petrova $\cdot$ Peter K. Petrov $\cdot$ Michael E. Edmonds • \\ Catherine M. Shanahan
}

Published online: 18 February 2014

(C) Springer Science+Business Media New York 2014

\section{Erratum to: Calcif Tissue Int \\ DOI 10.1007/s00223-013-9820-9}

Unfortunately, all reported values representing the area of resorption under the surface were missing the $10^{3}$ multiplication factor in abstract, surface profilometry section and Fig. 4 in the original publication. The correct presentation is shown in this erratum.

The eighth sentence of the abstract should read "The area of resorption on the surface by image analysis was significantly greater in Charcot patients compared with controls (21.1\% [14.5-26.2] versus $40.8 \%$ [35.4-46.0], median [25-75th percentile], $p<0.01$ ), as was the area of resorption under the surface $\left(2.7 \times 10^{3} \mu \mathrm{m}^{2}\left[1.6 \times 10^{3}-\right.\right.$ $\left.3.9 \times 10^{3}\right] \quad$ versus $8.3 \times 10^{3} \quad \mathrm{~mm}^{2} \quad\left[5.6 \times 10^{3}-\right.$ $\left.\left.10.6 \times 10^{3}\right], p<0.01\right)$ after profilometry".

The online version of the original article can be found under doi:10. 1007/s00223-013-9820-9.

N. L. Petrova $(\bowtie) \cdot$ M. E. Edmonds

Diabetic Foot Clinic, King's College Hospital, Denmark Hill,

London SE5 9RS, UK

e-mail: petrovanl@yahoo.com

M. E. Edmonds

e-mail: michael.edmonds@nhs.net

P. K. Petrov

Department of Materials, Imperial College, Prince Consort

Road, London SW7 2AZ, UK

e-mail: p.petrov@imperial.ac.uk

\section{M. Shanahan}

Cardiovascular Division, James Black Centre, King's College

London, 125 Coldharbour Lane, London SE5 9NU, UK

e-mail: cathy.shanahan@kcl.ac.uk
The last sentence of the fourth paragraph in the Results (section surface profilometry) should read "The total area of the resorbed bone under the surface was significantly different between control subjects $\left(2.7 \times 10^{3} \mu \mathrm{m}^{2}\left[1.6 \times 10^{3}-3.9\right.\right.$ $\left.\left.\times 10^{3}\right]\right)$ and Charcot patients $\left(8.3 \times 10^{3}{\mu \mathrm{m}^{2}}^{2}\left[5.6 \times 10^{3}-\right.\right.$ $\left.10.6 \times 10^{3}\right]$ ), and there was a threefold increase in the area of resorption under the surface in M-CSF + RANKL-treated cultures between Charcot patients and controls $(p<0.01)$ (Fig. 4b)".

The correct version of Fig. 4 is presented below. 
A
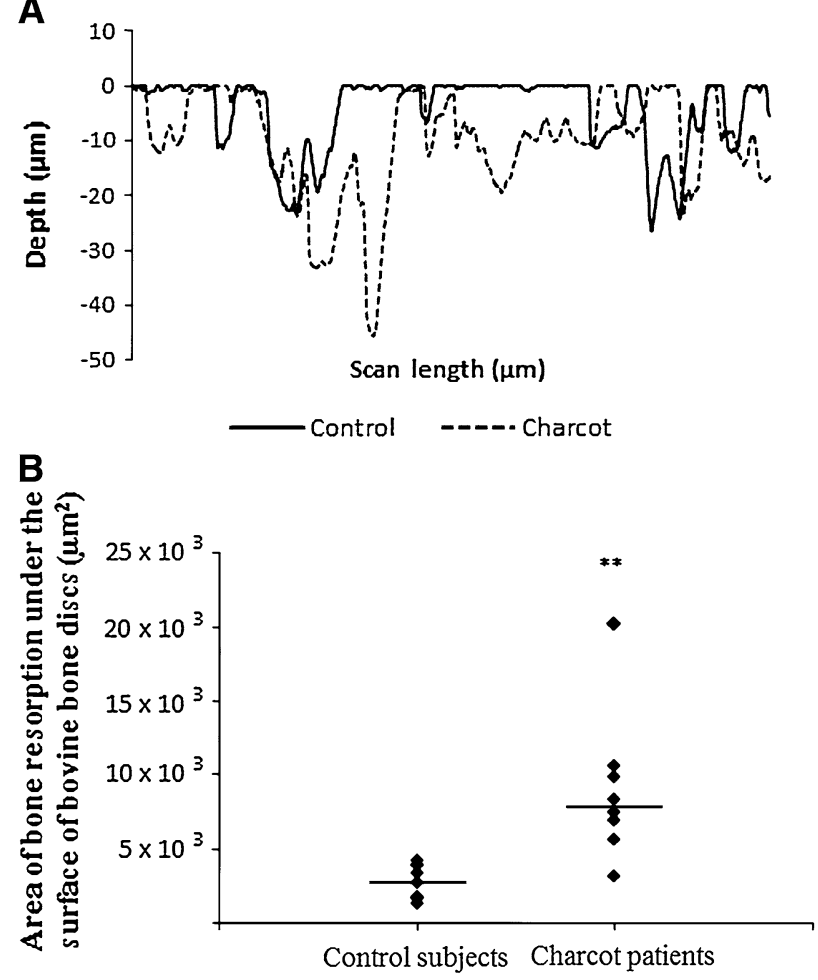

Fig. 4 Erosion profiles in a control subject (solid line) and a Charcot patient (dashed line) in M-CSF + RANKL-treated cultures after profilometry (a). Scatter plot graph represents the area of resorption under the surface on bovine bone discs $\left(\mu \mathrm{m}^{2}\right)$ in control subjects and Charcot patients in M-CSF + RANKL-treated cultures (b). Lines represent medians; $* * p<0.01$ 